\title{
SELECTED MEASUREMENTS OF RARE DECAYS AT THE LHCb EXPERIMENT*
}

\author{
Malgorzata Pikies \\ on behalf of the LHCb Collaboration \\ The Henryk Niewodniczański Institute of Nuclear Physics \\ Polish Academy of Sciences \\ Radzikowskiego 152, 31-342 Kraków, Poland
}

(Received April 6, 2016)

\begin{abstract}
Experimental results of rare decays $B^{0} \rightarrow K^{* 0} \mu \mu, B^{0} \rightarrow K^{* 0} e e, \Lambda_{b} \rightarrow$ $\Lambda \mu \mu$ and $B_{s}^{0} \rightarrow \phi \mu \mu$ governed by Flavour Changing Neutral Current transitions are discussed in this paper. The angular distributions and differential branching fractions measurements were performed using data corresponding to an integrated luminosity of $3.0 \mathrm{fb}^{-1}$ collected at the LHCb experiment.
\end{abstract}

DOI:10.5506/APhysPolB.47.1705

\section{Introduction}

The Large Hadron Collider beauty experiment $(\mathrm{LHCb})$ is located at the Large Hadron Collider (LHC). Currently, its main goal is to search for signals of physics beyond the Standard Model (SM), i.e. new physics.

The general scheme of the $\mathrm{LHCb}$ detector is shown in Fig. 1. It is a single arm forward spectrometer (covering the region of $2<\eta<5$ in units of pseudorapidity) with an excellent momentum resolution $\Delta p / p=0.5 \%$ at $5 \mathrm{GeV} / c$ to $0.6 \%$ at $100 \mathrm{GeV} / c$, an impact parameter resolution of $\sigma_{\mathrm{IP}} \sim$ $20 \mu \mathrm{m}$, a primary vertex resolution of $13 \mu \mathrm{m}$, in $x$ and $y$, and $71 \mu \mathrm{m}$ in $z$, a decay time resolution of $\sigma_{\tau} \sim 50$ fs and excellent particle identification system.

These and many other features made this detector a perfect instrument to study decays of particles containing beauty and charm quarks. The details of the detector can be found in Ref. [1].

* Presented at the Cracow Epiphany Conference on the Physics in LHC Run 2, Kraków, Poland, January 7-9, 2016. 


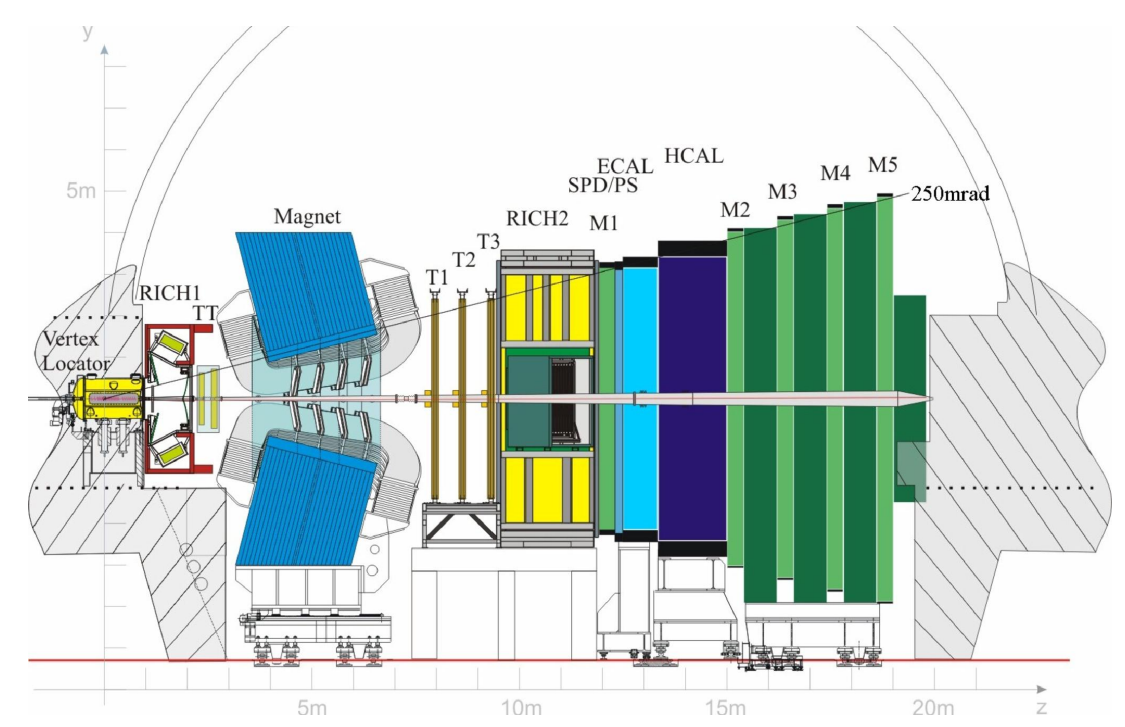

Fig. 1. View of the LHCb detector.

\subsection{The role of rare decays}

Flavour Changing Neutral Current (FCNC) transitions like $b \rightarrow s l l$, are only possible in the SM via loop processes. Two examples with two muons in the final state are shown in Fig. 2.
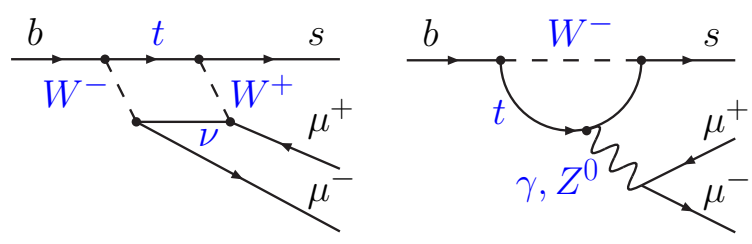

Fig. 2. The Standard Model $b \rightarrow s \mu \mu$ transitions for box (left) and penguin (right) diagrams.

In some extensions of the SM, new particles may enter these loops and, therefore, can significantly change the Lorentz structure of the vertex. In the following, the focus is turned to the selected electroweak penguin decays: $B^{0} \rightarrow K^{* 0} \mu \mu, B^{0} \rightarrow K^{* 0} e e, \Lambda_{b} \rightarrow \Lambda \mu \mu$ and $B_{s}^{0} \rightarrow \phi \mu \mu$.

\section{Selected $b \rightarrow$ sll transitions}

The decays discussed in this paper occur by the same type of $b$ to $s$ transitions. The effective Hamiltonian is given by 


$$
H_{\mathrm{eff}}=-\frac{4 G_{\mathrm{F}}}{\sqrt{2}} V_{t b} V_{t s}^{*} \sum_{i} C_{i} O_{i},
$$

where $C_{i}$ are short distance Wilson coefficients and $O_{i}$ are long distance Wilson operators.

\subsection{Angular analysis of the $B^{0} \rightarrow K^{* 0}\left(\rightarrow K^{+} \pi^{-}\right) \mu \mu$ decay}

Selected candidates were required to have a reconstructed invariant mass between $5170 \mathrm{MeV} / c^{2}$ and $5700 \mathrm{MeV} / c^{2}$ (Fig. 3). The reconstructed mass of the $K^{+} \pi^{-}$system was required to be in the range between $795 \mathrm{MeV} / \mathrm{c}^{2}$ and $966 \mathrm{MeV} / c^{2}$ [2], compatibile with $K^{*}$ mass.
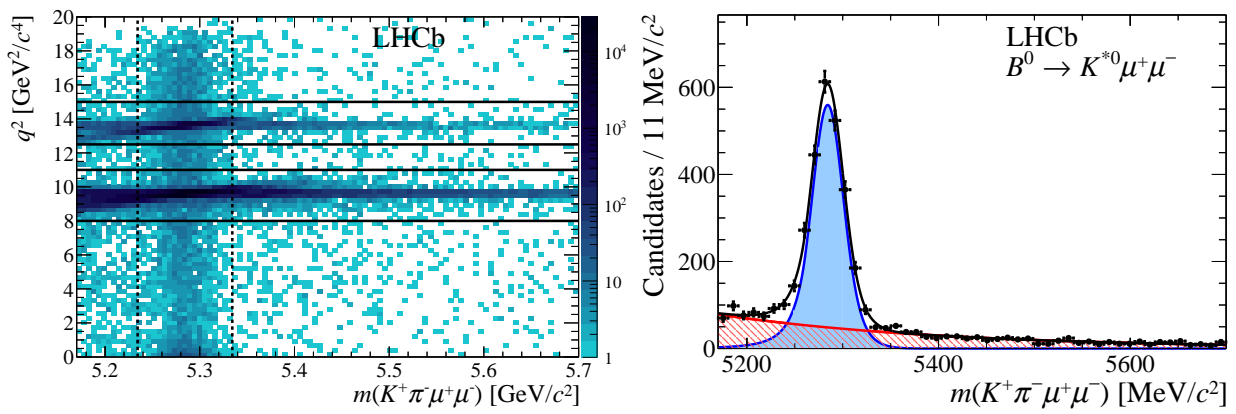

Fig. 3. Invariant mass distributions of the $K^{+} \pi^{-} \mu^{+} \mu^{-}$system versus the dimuon invariant mass $q^{2}$ (left) and integrated over $q^{2}$ (right).
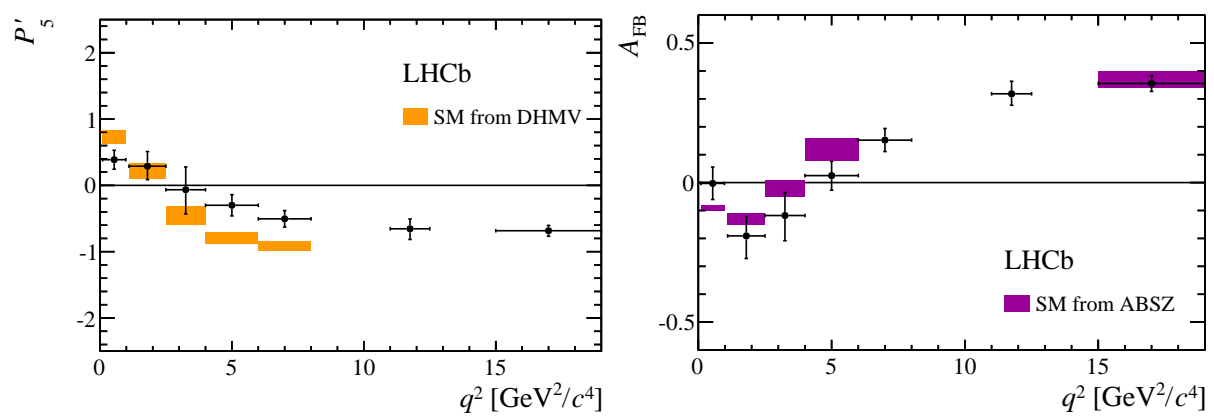

Fig. 4. Angular observables $P_{5}^{\prime}$ (left) and $A_{\mathrm{FB}}$ (right) as a function of $q^{2}$. Shadowed boxes show the SM predictions.

Combinatorial background was reduced using a Boosted Decision Tree trained on data, with $B^{0} \rightarrow J / \psi^{0} K^{* 0}$ decays as a signal proxy and candidates from an upper sideband as a background proxy. 
A recently updated analysis confirmed a local tension in the angular observable $S_{5}$ and also in the related distribution $P_{5}^{\prime}$ and in the forwardbackward asymmetry $A_{\mathrm{FB}}$ (Fig. 4) [2].

\subsection{Angular analysis of the $B^{0} \rightarrow K^{* 0}\left(\rightarrow K^{+} \pi^{-}\right)$ee decay}

The angular observables were measured for the first time in this decay channel [6]. The analysis was restricted to the low $q^{2}$ range $[0.002,1.120]$ $\mathrm{GeV}^{2} / c^{4}$ which is sensitive to the Wilson coefficient $C_{7}$, as can be seen in Fig. 5.

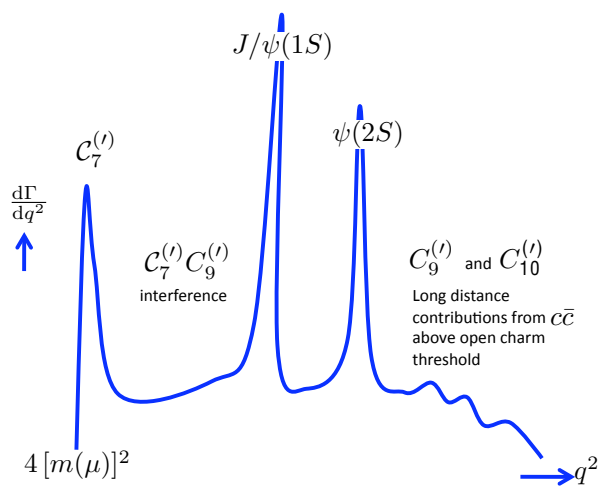

Fig. 5. Differential decay width as a function of $q^{2}$. The most relevant Wilson coefficient is superimposed.

A total of 124 signal events passed the selection in the $B^{0}$ mass window [4800-5400] MeV $/ c^{2}$ as shown in Fig. 6. The angular observables obtained from the fit show good agreement with the SM predictions.

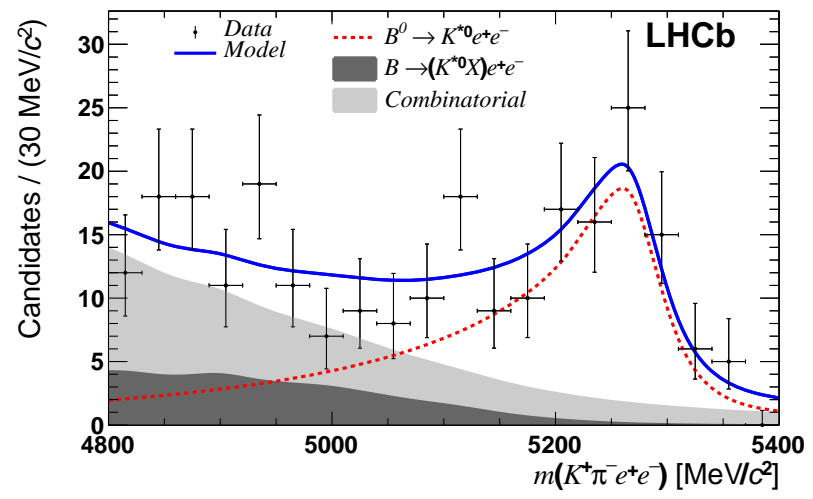

Fig. 6. Invariant-mass distribution of the $K^{+} \pi^{-} e^{+} e^{-}$candidates. 


\subsection{Angular analysis and differential branching fraction measurement of the $\Lambda_{b} \rightarrow \Lambda\left(\rightarrow p \pi^{-}\right) \mu \mu$ decay}

The differential branching fraction was measured as a function of $q^{2}$. The signal decay $\Lambda_{b} \rightarrow \Lambda \mu \mu$ was normalized to a $\Lambda_{b} \rightarrow \Lambda J / \psi$ channel. Figure 7 shows that the measured differential branching fraction as a function of $q^{2}$ is consistent with the SM in the high- $q^{2}$ region, but lays below the SM prediction in the low- $q^{2}$ region $[7,8]$.

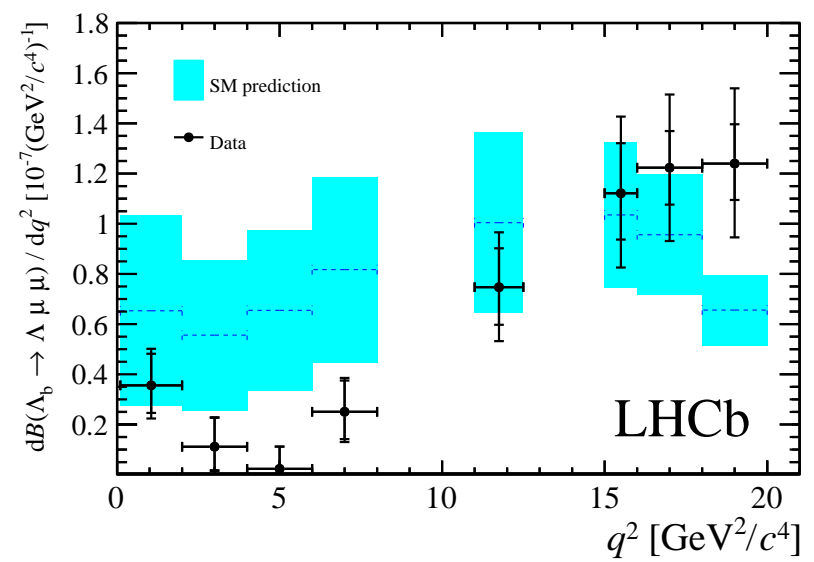

Fig. 7. Measured $\Lambda_{b} \rightarrow \Lambda \mu \mu$ differential branching fraction as a function of $q^{2}$. Shadowed boxes show the SM predictions.

The angular analysis was made for leptonic $\left(A_{\mathrm{FB}}^{l}\right)$ and hadronic $\left(A_{\mathrm{FB}}^{h}\right)$ forward-backward asymmetries. The $A_{\mathrm{FB}}^{l}$ measurements showed deviations from SM predictions in the high- $q^{2}$ region as can be seen in Fig. 8 [9].
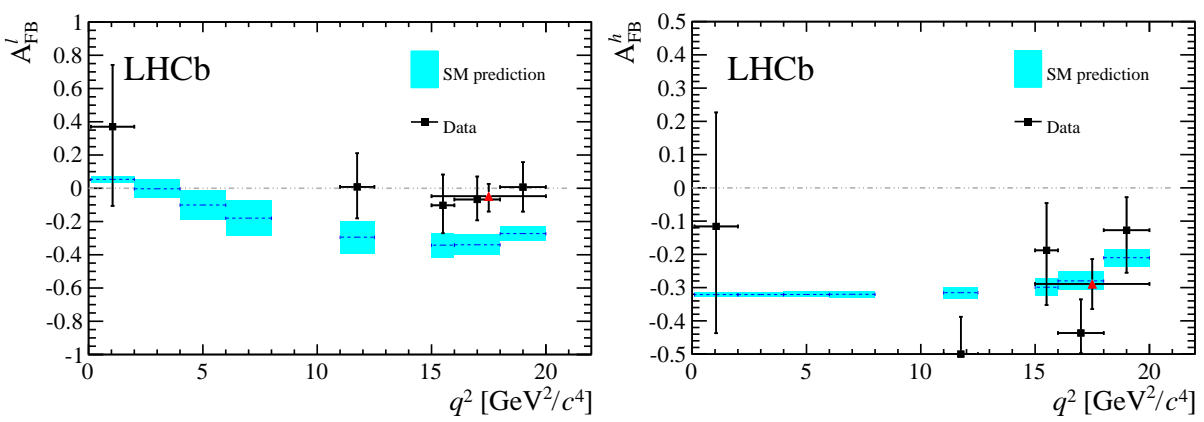

Fig. 8. Forward-backward asymmetries of the leptonic (left) and hadronic (right) components as a function of $q^{2}$. Shadowed boxes show the SM predictions. 


\subsection{Angular analysis and differential branching fraction measurement of $B_{s}^{0} \rightarrow \phi\left(\rightarrow K^{+} K^{-}\right) \mu \mu$ decays}

This decay is similar to the $B^{0} \rightarrow K^{* 0} \mu \mu$ transition but the final state is not flavour-specific, hence the accessible observables are $F_{\mathrm{L}}, S_{3,4,7}, A_{5,6,8,9}$. The angular analysis shows good agreement with the SM but the differential branching fraction measurements in the low- $q^{2}$ region, where good theoretical predictions are accessible, differs from the SM by $3.3 \sigma$ as can be seen in Fig. $9[5,10,11]$.

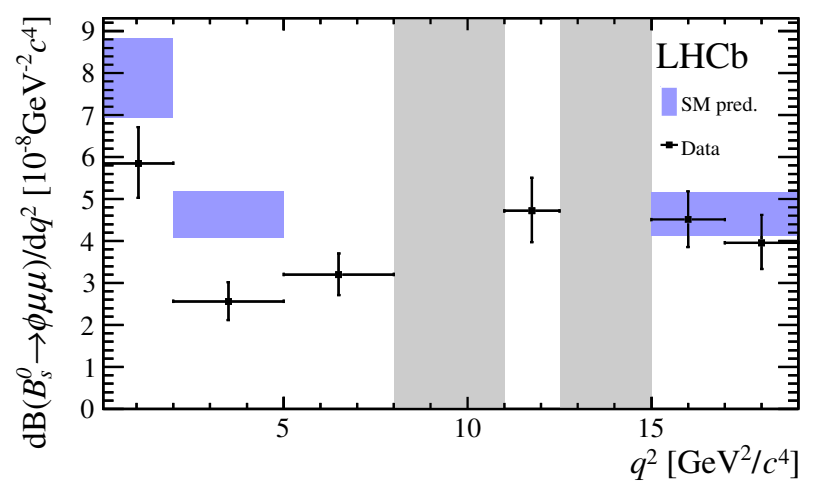

Fig. 9. Differential branching fraction of the decay $B_{s}^{0} \rightarrow \phi \mu \mu$. Shadowed boxes show the SM predictions.

\section{Summary}

Rare decays are a powerful tool for searching indirect evidence of new physics. During Run 1, the LHCb experiment collected data corresponding to $3.0 \mathrm{fb}^{-1}$ of integrated luminosity. A number of analyses dedicated to the family of $b \rightarrow s$ transitions showed tensions with the Standard Model predictions in differential branching fraction measurements as a function of $q^{2}$. Furthermore, the LHCb golden channel $B^{0} \rightarrow K^{* 0} \mu \mu$ showed tension in angular observables.

I would like to express my gratitude to the National Science Centre (NCN) in Poland for financial support under the contract No. 2015/17/D/ $\mathrm{ST} 2 / 03532$. 


\section{REFERENCES}

[1] R. Aaij et al. [LHCb Collaboration], Int. J. Mod. Phys. A 30, 1530022 (2015).

[2] R. Aaij et al. [LHCb Collaboration], CERN-PH-EP-2015-314;

LHCB-PAPER-2015-051

[3] J. Matias, F. Mescia, M. Ramon, J. Virto, J. High Energy Phys. 1204, 104 (2012).

[4] S. Descotes-Genon, L. Hofer, J. Matias, J. Virto, J. High Energy Phys. 1412,125 (2014).

[5] W. Altmannshofer, D. Straub, Eur. Phys. J. C 75, 382 (2015).

[6] R. Aaij et al. [LHCb Collaboration], J. High Energy Phys. 1504, 64 (2015).

[7] R. Aaij et al. [LHCb Collaboration], J. High Energy Phys. 1506, 115 (2015).

[8] W. Detmold, C.-J.D. Lin, S. Meinel, M. Wingate, Phys. Rev. D 87, 074502 (2013).

[9] S. Meinel, PoS LATTICE2013, 024 (2013).

[10] A. Bharucha, D. Straub, R. Zwicky, TUM-HEP-957/14 CP3-Origins-2015-010 DNRF90 DIAS-2015-10.

[11] R. Aaij et al. [LHCb Collaboration], J. High Energy Phys. 1509, 179 (2015). 